\title{
CT Brain Perfusion Protocol to Eliminate the Need for Selecting a Venous Output Function
}

\author{
A.J. Riordan, E. Bennink, M.A. Viergever, B.K. Velthuis, J.W. Dankbaar, and H.W.A.M. de Jong
}

\section{ABSTRACT}

BACKGROUND AND PURPOSE: In CTP, an arterial input function is used for cerebral blood volume measurement. AIFs are often influenced by partial volume effects resulting in overestimated CBV. A venous output function is manually selected to correct for partial volume. This can introduce variability. Our goal was to develop a CTP protocol that enables AIF selection unaffected by partial volume.

MATERIALS AND METHODS: First, the effects of partial volume on artery sizes/types including the MCA were estimated by using a CTP phantom with 9 protocols (section thicknesses of $1,1.8$, and $5 \mathrm{~mm}$ and image resolutions of $0.5,1$, and $1.5 \mathrm{~mm}$ ). Next, these protocols were applied to clinical CTP studies from 6 patients. The influence of the partial volume effect was measured by comparison of the timeattenuation curves from different artery locations with reference veins.

RESULTS: AIFs from MCAs were unaffected by partial volume effects when using high image resolution (1 mm) and medium section thickness $(1.8 \mathrm{~mm})$. For the clinical data, a total of 104 arteries and 60 veins was selected. The data confirmed that high image resolution and thin section thickness enable selection of MCAs for AlFs free of partial volume influences. In addition, we found that large veins were not insusceptible to partial volume effects relative to large arteries, questioning the use of veins for partial volume correction.

CONCLUSIONS: A CTP protocol with 1.8-mm section thickness and 1-mm image resolution allows AIF selection unaffected by partial volume effects in MCAs.

ABBREVIATIONS: AIF = arterial input function; $\mathrm{AUC}=$ area under the curve; $\mathrm{PVE}=$ partial volume effect; $\mathrm{VOF}=$ venous output function; $\mathrm{SRT}=$ signed rank test

TP is used in the diagnosis of acute ischemic stroke to noninvasively measure cerebral perfusion parameters. ${ }^{1-5}$ Relative perfusion parameters can be obtained by normalization to the uninvolved hemisphere and are successfully applied in stroke imaging to differentiate penumbra from benign oligemia. ${ }^{6-8} \mathrm{Accu}-$ rate absolute CTP values would be desirable to improve the comparability of results. As an example, absolute CBV has been found to be an accurate method of delineating the infarct core, ${ }^{9}$ and this method is widely used in clinical practice, providing motivation to assess and improve the accuracy of CBV measurements.

Calculation of CTP maps requires selection of an arterial input function (Fig 1). The AUC (the integral of contrast agent concentration with time) of the AIF is used to calculate the CBV. However, this AUC is commonly underestimated due to

Received July 20, 2012; accepted after revision September 24.

From the Department of Radiology, University Medical Center Utrecht, Utrecht, the Netherlands.

Please address correspondence to A. Riordan, MD, Department of Radiology, University Medical Center Utrecht, Room E02.222, Heidelberglaan 100, 3584 CX

Utrecht, the Netherlands; e-mail: A.J.Riordan@umcutrecht.nl

http://dx.doi.org/10.3174/ajnr.A3397 the partial volume effect, especially when smaller arteries like the anterior cerebral artery or MCA are selected. The PVE is a result of limited spatial resolution as defined by the in-plane axial image resolution and section thickness. ${ }^{10-13}$ Because underestimation of the AUC of the AIF results in overestimation of the CBV, correction for PVE is necessary and typically performed by using the VOF. ${ }^{14-20}$ This correction assumes that without PVE, all AIFs and VOFs should have equal AUCs and that a VOF selected in a large vein like the superior sagittal sinus or the straight sinus is unaffected by PVE.

Correction of the PVE by using the VOF has limitations. It requires the user to select a second vessel besides the AIF, introducing additional user variability. Also, the AUC of the VOF has been reported to depend on section thickness ${ }^{17}$ and cannot always be considered uninfluenced by PVE. Finally, although the assumption of equal AUCs in AIF and VOF is plausible, it has not been investigated so far, to our knowledge.

The goal of this study was to develop a CTP protocol that would enable PVE-free AIF selection in the MCA by increasing image resolution and decreasing section thickness, eliminating a step that every clinician must currently make in perfusion analysis.

AJNR Am J Neuroradiol 34:1353-58 Jul 2013 www.ajnr.org

1353 


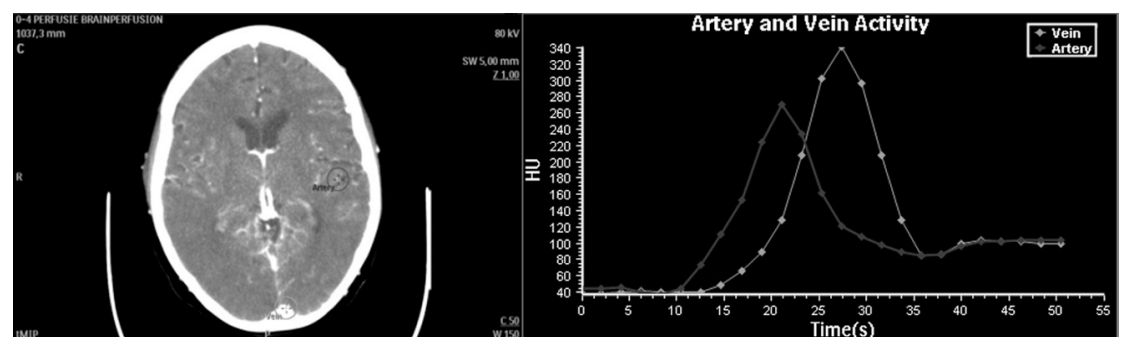

FIG 1. Semiautomatic blood vessel designation requires user definition of ROls (circles) on an artery and a large vein. The software then determines the voxel within the region of interest representing the time-attenuation curve with maximum AUC.

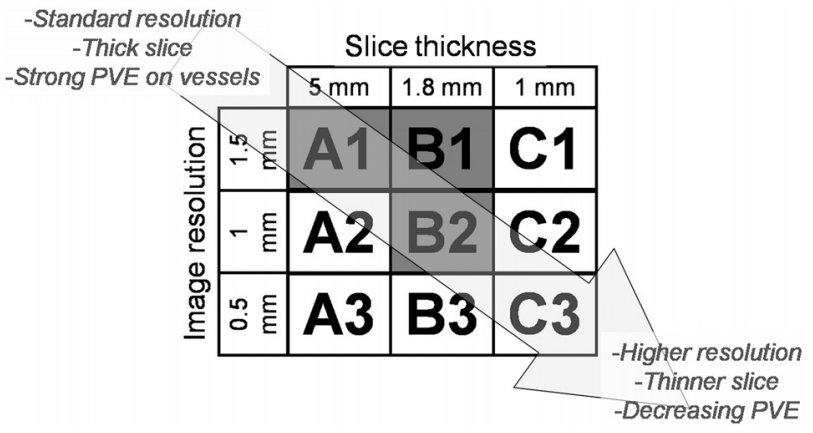

FIG 2. CTP protocols investigated by using the head phantom. Protocols $\mathrm{A} 1, \mathrm{~B} 1$, and $\mathrm{B} 2$ have also been used in acquiring the clinical data.

\section{MATERIALS AND METHODS}

The required section thickness and image resolution were established by using phantom and patient studies.

\section{Anthropomorphic Head Phantom}

A realistic anthropomorphic CTP head phantom, previously used to compare CTP software and protocols, ${ }^{21}$ was applied to gain insight into the PVE for a range of CTP reconstruction protocols. The phantom also allows measurement of PVE with knowledge of the underlying truth. The CTP phantom is a combination of CT images (using the Brilliance iCT 256 [Philips Healthcare, Best, the Netherlands] section protocol, detailed in the "Clinical Data" section) of a physical skull phantom together with brain MR images. Spatially variant CT noise, effects of tube settings, and reconstruction parameters are included by scanning the skull phantom by using the CTP protocol. Cerebral vessels and tissue, automatically segmented from the MR imaging data, ${ }^{22,23}$ were manually registered to fit inside CT images of the skull phantom. Time-attenuation curves in the arteries and veins (AIF and VOF) were taken from clinical scans. Arterial and venous time-attenuation curves from clinical data were added, and perfusion values observed in clinical studies were used to generate tissue time-attenuation curves. Pixel size and section thickness can be set by averaging the original image voxels and resolution by changing the scale of a blurring kernel.

Phantom data were constructed by using 9 different protocols (Fig 2), varying in section thickness and in section image resolution. These parameters mainly define the $3 \mathrm{D}$ spatial resolution of the CTP images and hence the strength of the PVE for a vessel. ${ }^{24}$ The section thicknesses used were the following: $1 \mathrm{~mm}$, achievable with most modern scanners; $1.8 \mathrm{~mm}$, and $5 \mathrm{~mm}$, often used in
CTP protocols to reduce noise in the tissue time-attenuation curves.

The in-plane image resolution depends on several parameters, including focal spot size, image reconstruction filter, and pixel size, which can be set by the operator. The image resolution was expressed in the full width at half maximum (in millimeters) of the point spread function and was measured by using an imagequality phantom. For the head phantom, we choose image resolutions of $0.5 \mathrm{~mm}$, a typical technical limit for most scanners, and 1 and $1.5 \mathrm{~mm}$, typical of current CTP protocols. In all cases, the pixel size was set to $0.2 \times 0.2 \mathrm{~mm}$. To ensure that variance in the measured PVE could not be attributed to noise, the phantoms were constructed by using unrealistically high milliampere-second, approximately 100 times higher than that used in a clinical setting.

\section{Clinical Data}

An initial group of 30 patients was selected from participants in the Dutch Acute Stroke Trial (http://clinicaltrials.gov/ct2/show/ NCT00880113), a national multicenter prospective study on the prognostic value of CTP and CTA in patients with acute stroke symptoms. This study was approved by the institutional review committee, and subjects gave informed consent. Inclusion criteria were as follows: 1) patients who displayed no hemorrhage or infarcted tissue on both admission and 3-day follow-up noncontrast CT, to ensure that all of the contrast agent entering via the arteries would exit via the veins and that all time-attenuation curves were fully sampled; 2) admission scan on a 256-section scanner, to ensure a uniform quality of data; 3) CTP acquisition in thin-section mode to allow reconstruction of different section thicknesses. Next, the patients were visually assessed by an expert reader to ensure the absence of any motion and that the ICA and MCA were present, resulting in 24 of these patients being eliminated; the 6 patients left were included in this study.

All data were acquired and constructed by using the standard clinical protocol: $150 \mathrm{mAs} /$ rotation, $80 \mathrm{kV}$ (peak), $512 \times 512 \mathrm{ma}-$ trix, 200-mm FOV, 0.39- $\mathrm{mm}^{2}$ pixels, section thickness/spacing of $1 / 0.8 \mathrm{~mm}$, and an acquisition series of 25 time frames at 2-second intervals with a rotation time of 0.33 seconds. These data were subsequently reconstructed to a section thickness of $5 \mathrm{~mm}$ (clinical standard) or $1.8 \mathrm{~mm}$ (thin section) and an in-plane image resolution (point spread function) of $1.5 \mathrm{~mm}$ (clinical standard) or $1 \mathrm{~mm}$ (high resolution) in the combinations given in Fig 2 .

\section{Image Analysis}

Both the phantom data and the clinical data were analyzed in a similar fashion. Circular regions of interest with diameters of 20 $\mathrm{mm}$, to ensure complete encircling of the vessel cross-section, were manually drawn over artery locations in the maximum intensity projections (Fig 1). These arteries were approximately perpendicular to the section plane. ${ }^{17}$ The content of each region of interest in the raw data was examined to determine the artery diameter and center. The diameter and center were estimated by using a threshold technique, ${ }^{25}$ allowing grouping in artery types: 

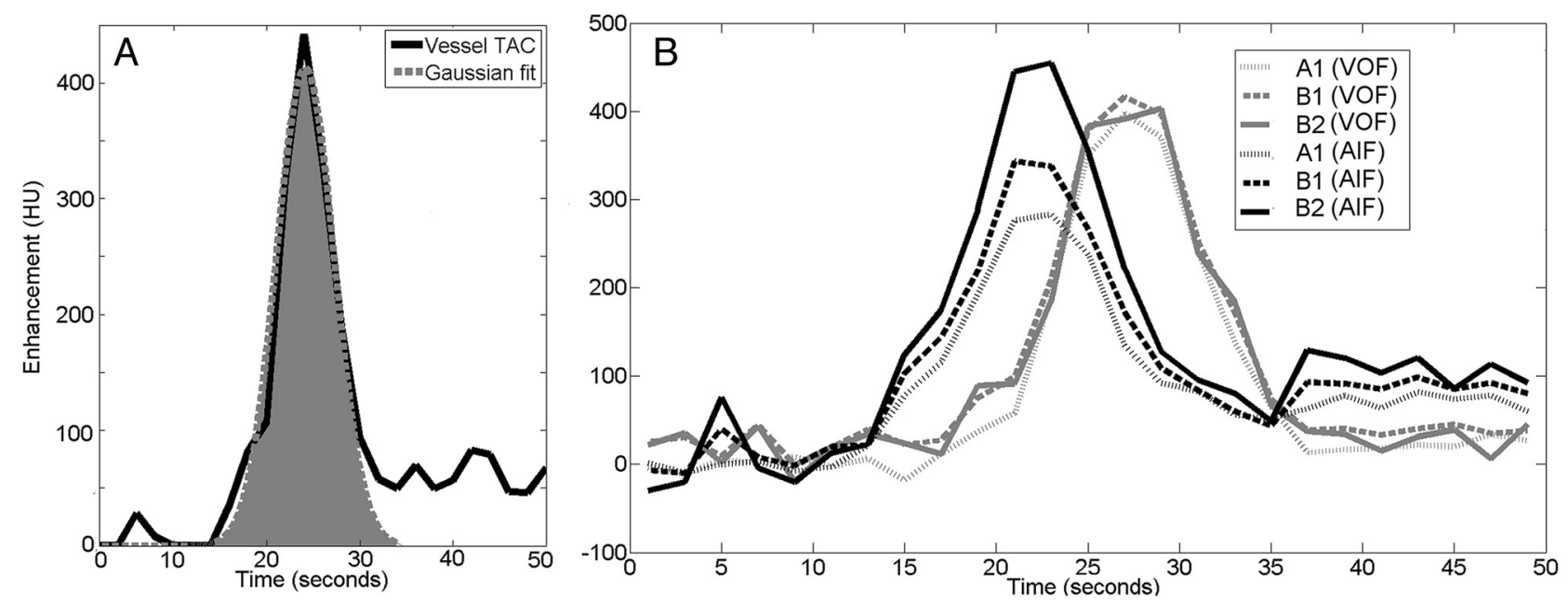

FIG 3. $A$, Gaussian fit to the AUC (shaded area) of a vessel to estimate the AUC of the first pass of the bolus. $B$, Time-attenuation curves, background-corrected, demonstrate the changes in enhancement for the different protocols, A1, B1, and B2. Black lines represent an MCA, and gray lines, a vein from the same patient. The MCA has a higher AUC than the vein when using protocol B2.

ICA (diameter $\geq 4 \mathrm{~mm})$, MCA $(2-3 \mathrm{~mm})$, and smaller arteries (1-2 mm) such as small MCA and anterior cerebral artery vessels. This technique proved accurate as long as the vessel diameter was not smaller than the full width at half maximum of the CT images. ${ }^{25}$ Selection of the VOF was similar to the selection of the AIF, though now the ROIs were placed over large veins (sagittal sinus) that were approximately perpendicular to the imaging plane.

Because the vessel center can be considered least affected by partial volume, the AUC was estimated by fitting a Gaussian curve to the time-attenuation curve of the pixel determined to be at the center. Only the center pixel was measured to conform to methods used in clinical perfusion software and to ensure that the PVE was not overestimated in smaller vessels by averaging with pixels nearer to the vessel wall. The Gaussian curve fit enabled us to distinguish the first-pass bolus from the recirculation as is done in commercial software such as Brain Perfusion 4.0 (Philips Healthcare) (Fig 3A). Before curve fitting, time-attenuation curves were corrected for background, defined as the local time-averaged Hounsfield unit value before bolus arrival.

A measure for the PVE in the vessels was calculated by dividing the AUC of the AIF by the AUC of a reference time-attenuation curve, yielding a relative AUC $\left(\mathrm{AUC}_{\mathrm{rel}}\right)$. For the phantom data, the known input arterial time-attenuation curve before PVE influences served as the reference time-attenuation curve. For the clinical data, the VOF measured with protocol B1 (Fig 2) was used as the reference. $\mathrm{AUC}_{\text {rel }}$ values were expected to be $\leq 1$, where 1 could be interpreted as being unaffected by PVE. Values of $<1$ could be considered as a PVE-induced reduction of the AUC. The relative value of 1 was a reference point so that patients with differing enhancement may be compared. Ideally, we wished to find the vessel with the highest enhancement, and $\mathrm{AUC}_{\mathrm{rel}}$ values of $>1$ were allowed for in the clinical data, for example, if an artery had a higher AUC than the reference vein. The results from the clinical data were grouped according to vessel type (small arteries, MCA, ICA, and large veins) and protocol used.

\section{Statistical Analysis}

Statistical analyses by using the Statistical Package for the Social Sciences software (SPSS, Chicago, Illinois) determined the significance and implication of the clinical measurements. Depending on the relative group size, relationship, and the number of groups being compared, either a Mann-Whitney 2-tailed, Friedman with post hoc, or Wilcoxon signed rank test was used to test the difference in PVE results between vessel groups and CTP protocols. In all cases, probability values are reported, and a $P$ value of .05 was considered significant in all test types.

\section{RESULTS}

\section{Anthropomorphic Head Phantom}

Figure 4 shows that the protocol with a section thickness of $\leq 1.8$ $\mathrm{mm}$ and an image resolution of, at most, $1 \mathrm{~mm}$ (protocol B2) shows the least PVE for the MCA vessel group. In the boxplot, $\mathrm{AUC}_{\text {rel }}$ values are compared among the 9 CTP protocols. The impact of PVE reduces with higher (improved) image resolution and thinner sections (protocols B and C).

\section{Clinical Data}

From the 6 patient studies, a total of 104 AIF and 60 VOF locations were selected. At every AIF and VOF location, the time-attenuation curve was measured by using the 3 protocols, A1, B1, and B2. Examples of MCA and venous time-attenuation curves are shown in Fig $3 B$. The time-attenuation curve of the AIF gets higher with smaller section thickness and higher (improved) resolution. This effect is much less pronounced or even absent for the venous time-attenuation curves.

Figure 5, a boxplot, shows the median and range of $\mathrm{AUC}_{\mathrm{rel}}$ for the 3 artery groups and the large veins. In all vessel groups, the protocols with smaller section thickness and higher resolution, in the order of A1, B1, and B2, show decreasing PVEs. This boxplot merely illustrates the distribution of the samples; the Table contains statistical analysis that investigates the trends observable in this figure.

For the smaller arteries, $\mathrm{AUC}_{\text {rel }}$ stays well below 1 for all eval-

AJNR Am J Neuroradiol 34:1353-58 Jul 2013 www.ajnr.org

1355 


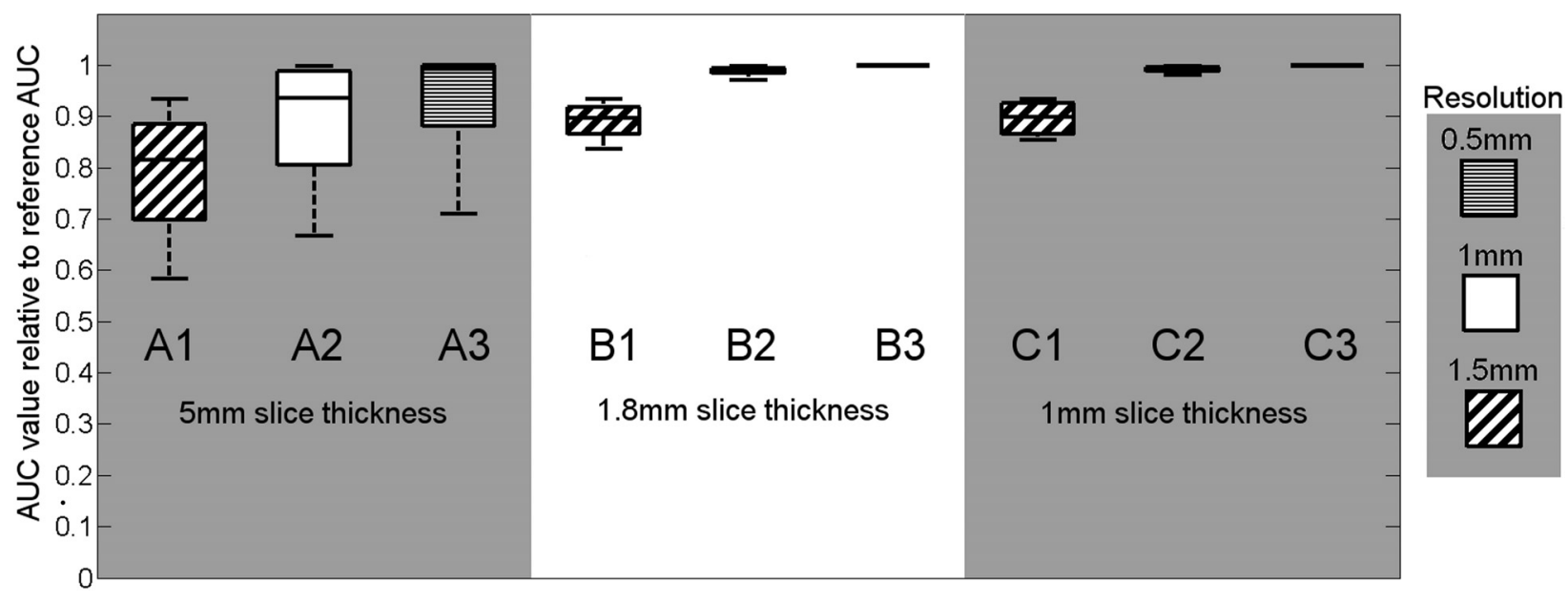

FIG 4. Results of PVE measurements applying 9 different CTP protocols to the digital head phantom. The vertical axis gives a measure of the partial volume effect expressed as $A \cup C_{\text {rel }}$.

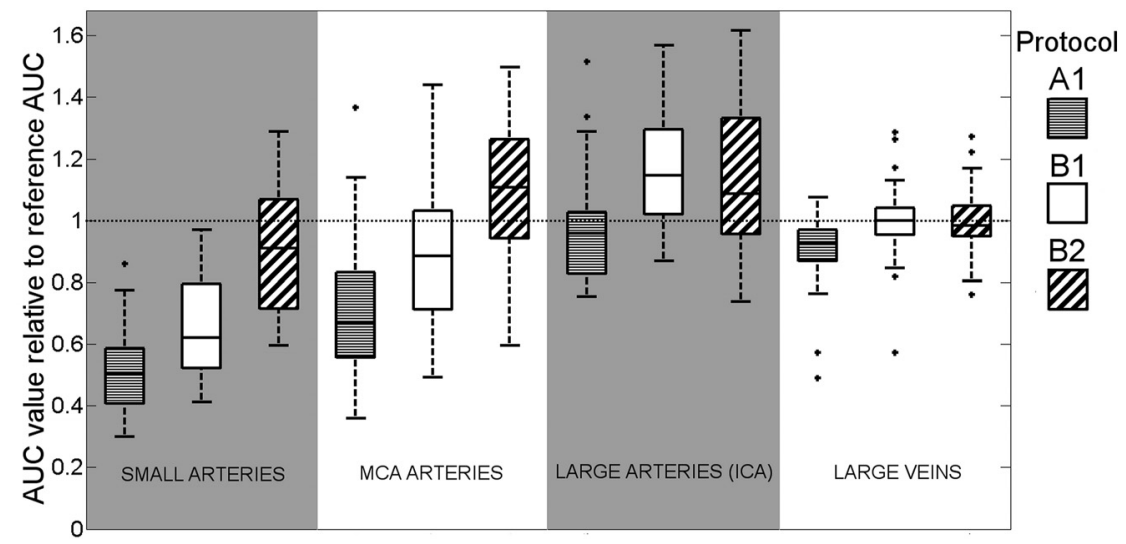

FIG 5. Boxplot describing how PVE depends on CTP protocol. Vessel type and group are defined on the horizontal axis; AUC of the vessel time-attenuation curve relative to $A \cup C$ of the average VOF from protocol $B 1$ ( $A \cup C_{\text {rel }}$ ), as a measure for partial volume effect is displayed on the vertical axis. Each of the groupings represents a range of vessel diameters (small arteries, $<2 \mathrm{~mm}$; MCA, 2-3 mm; large arteries [ICA], $>4 \mathrm{~mm}$; vein, $>5 \mathrm{~mm})$.

Summary of the statistical results and implications obtained by comparison of the vessel AUC measured in the clinical data ${ }^{a}$

\begin{tabular}{|c|c|c|c|}
\hline Comparison & Test Applied & $P$ Value & Implication \\
\hline Vein $B 1$ vs vein $B 2$ & Wilcoxon SRT & .941 & No difference in VOFs between protocols $\mathrm{B} 1$ and $\mathrm{B} 2$ \\
\hline Vein $A 1$ vs vein $B 2$ & Wilcoxon SRT & $<.001$ & $\begin{array}{l}\text { VOFs significantly different (stronger PVE) with clinical standard } \\
\text { protocol Al compared with protocol B2 }\end{array}$ \\
\hline ICA B1 vs ICA B2 & Wilcoxon SRT & .211 & No significant difference in ICA AIF between protocols B1 and B2 \\
\hline ICA AI vs ICA BI & Wilcoxon SRT & $<.001$ & $\begin{array}{l}\text { ICA AIFs significantly different (stronger PVE) with clinical standard } \\
\text { Al compared with protocol BI }\end{array}$ \\
\hline MCA A1 vs MCA B1, B2 & Friedman & $<.001$ & AIFs in MCA significantly different in all protocols \\
\hline Small A1 vs small B1, B2 & Friedman & $<.001$ & AIFs in small vessels significantly different in all protocols \\
\hline MCA B2 vs ICA B2 & Mann-Whitney & .519 & $\begin{array}{l}\text { No significant difference in MCA and ICA when protocol B2 is } \\
\text { applied }\end{array}$ \\
\hline ICA A1, A2, B2 & Mann-Whitney & $<.001$ & VOF significantly lower than ICA for all protocols \\
\hline \multicolumn{4}{|l|}{ VOF A1, A2, B2 } \\
\hline Clinical results vs phantom results & Mann-Whitney & .49 & PVE results from clinical data correlate with head phantom \\
\hline
\end{tabular}

${ }^{a}$ A $P$ value of .05 was considered significant for all tests.

uated protocols. For the veins, the average $\mathrm{AUC}_{\mathrm{rel}}$ is 1 , by definition, for protocol B1 and approximately 1 if B2 is used. When applying protocol A1 (often used clinically) with a 5-mm section thickness, the veins are still slightly affected by PVE. This finding is important, given that in clinical practice, PVE correction is commonly based on the VOF.
For the larger arteries, the MCA group showed less PVE for protocol B2 over B1. For the ICA, protocols B1 and B2 showed similar results, which indicates that for large arteries, protocol B1 suffices to allow AIF selection unaffected by PVE in the MCA. Most interesting, the larger artery (MCA and ICA) groups showed an $\mathrm{AUC}_{\text {rel }}$ larger than 1 . This is unexpected because current CTP 
methodology assumes the AUC of the arteries to be ideally equal to that of the veins.

The Table shows a summary of the statistical comparisons that confirm the significance of the results. Specific tests, Wilcoxon SRT, Friedman, and Mann-Whitney, were performed in consultation with a trained statistician, taking into consideration the asymmetry of the distributions, number of samples per group, and number of groups being compared. The implications will be examined further in the "Discussion."

\section{DISCUSSION}

Although previous studies ${ }^{17,26}$ have investigated PVE under different CT protocols and conditions, there are no published data on what protocol would eliminate the need for correction of AIF measurements. In addition, there are no studies that have validated correction of PVE by using the measurements of the VOF, to our knowledge. The results of our phantom experiments indicated that an image resolution of $1 \mathrm{~mm}$ and a section thickness of $1.8 \mathrm{~mm}$ enable AIF definition in MCA vessels unaffected by PVE. The application of this protocol to clinical data resulted in good agreement with the findings from the phantom study, and the area under the curve of the MCA was found to be larger or equal to the VOF.

The spread in the measured values from the protocols A1-A3 (Fig 4) is mainly attributed to variance in the vessel orientation between approximately $0^{\circ}$ and $30^{\circ}$, which has a substantial influence when using thick sections. While it is possible to reconstruct CTP volumes with a section thickness limited only by the hardware $(0.625 \mathrm{~mm}$ in the case of the scanner used here), the time taken to process $>100$ sections for a 7 - to 8 -cm-long CTP image volume can be prohibitive for a time-critical procedure, in which a quick diagnosis is crucial. Reconstructing to 1.8 -mm-thick sections is more practical (protocols B1-B3), and the phantom results indicate that thinner sections (protocol C1-C3) offer no additional benefit. For the smaller arteries, the $\mathrm{AUC}_{\text {rel }}$ stayed well below 1 for all evaluated protocols, indicating that these vessels are not useable for AIF selection if PVE is to be prevented.

The measurements performed on the clinical data (Fig 5) have a noticeable variance. This can largely be attributed to uncertainty in vessel diameter measurements and vessel orientation and is illustrated by the fact that the large veins, where vessel diameter and orientation are less of an issue, have the least variance.

The unexpected finding that for the high-resolution protocols, the AUC of arteries was significantly larger than that of the veins (Table 1) is contrary to the common assumption that both AUCs are equal in the absence of PVE and hence can be used for PVE correction. In this study, every care was taken so that this difference could not be attributed to biases induced by curve fitting, background correction, delay of venous peak (the Gaussian fit is insensitive to delay), noise, or other image-related sources. This suggests that the PVE correction by using the VOF will not be sufficient but will still result in overestimation of the CBV.

It has previously been observed that the hematocrit in arteries and veins can differ. ${ }^{27,28}$ It is also well-documented that the high osmolarity of CT contrast agents, which acutely increases the blood volume, causes red blood cell crenation. ${ }^{29}$ It has been theorized that this could further change the hematocrit value in such a way that it is dependent on the size of the red blood cells and the pressure in the vessel, both of which differ between the arteries and veins. ${ }^{27}$ This theory implies that it might be incorrect to assume that the hematocrit factor is the same in the veins and arteries and that the relative hematocrit is dynamic due to the changing concentration of contrast in the blood.

Several limitations to this study must be acknowledged. First, 6 patients are a small cohort: The inclusion criteria were stringent to minimize the influence of confounding factors. In this study, we focused on the PVE, keeping all other aspects of CTP studies as constant as possible. The total number of included vessels enabled us to draw statistically significant conclusions. Second, when working with the suggested thin-section data, CT noise may affect CTP map quality, because tissue time-attenuation curves have lower contrast enhancement compared to vessel time-attenuation curves. It may be necessary to average the thin sections to thicker sections after acquisition of the AIF, to reduce noise in the tissue time-attenuation curves.

An alternative method to prevent loss of valuable time may be to reconstruct a limited number of thin sections purely for the acquisition of the AIF and to reconstruct the rest of the volume in thicker sections. In this study, care was taken to minimize the effect of noise. The vessel center was chosen as the measurement location. One alternative was to measure the pixel with the maximum enhancement; however, this measurement may have noiseinduced bias toward higher AUC values and influences the calculation of the PVE. In addition, the contrast enhancement in the vessels was high in comparison with the background noise in all protocols, allowing for constantly good fitting of the Gaussian shape to the first pass of the bolus. Because the method used to measure the AUC in this work is very similar, if not identical, to that in commercial CTP software, the measurements will reflect any bias observed in clinical practice.

\section{CONCLUSIONS}

Partial volume effects depend strongly on scan parameters and vessel diameter and can adversely affect CTP results. CTP data reconstructed with 1.8- $\mathrm{mm}$ section thickness and an image resolution of $1 \mathrm{~mm}$ can eliminate PVEs in MCA-sized arteries, which are found in all CTP scans. This protocol makes CTP scans less user-variant because no VOF selection is required. Such a reconstruction protocol may increase the accuracy of CTP maps and its subsequent diagnostic value.

Disclosures: Max A. Viergever-UNRELATED: Grants/Grants Pending: Philips Healthcare. * Birgitta K. Velthuis-Payment for Lectures (including service on Speakers Bureaus): CT perfusion presentations for Philips Healthcare. * *Money paid to the institution.

\section{REFERENCES}

1. Klotz E, Konig M. Perfusion measurements of the brain: using dynamic CT for the quantitative assessment of cerebral ischemia in acute stroke. Eur J Radiol 1999;30:170-84

2. Mayer TE, Hamann GF, Baranczyk J, et al. Dynamic CT perfusion imaging of acute stroke. AJNR Am J Neuroradiol 2000;21:1441-49

3. Wintermark M, Smith WS, Ko NU, et al. Dynamic perfusion CT: optimizing the temporal resolution and contrast volume for calculation of perfusion CT parameters in stroke patients. AJNR Am J Neuroradiol 2004;25:720-29

AJNR Am J Neuroradiol 34:1353-58 Jul 2013 www.ajnr.org 
4. Axel L. Cerebral blood flow determination by rapid-sequence computed tomography: theoretical analysis. Radiology 1980;137: 679-86

5. Axel L. A method of calculating brain blood flow with a CT dynamic scanner. Adv Neurol 1981;30:67-71

6. Lev MH, Segal AZ, Farkas J, et al. Utility of perfusion-weighted CT imaging in acute middle cerebral artery stroke treated with intraarterial thrombolysis: prediction of final infarct volume and clinical outcome. Stroke 2001;32:2021-28

7. Schaefer PW, Barak ER, Kamalian S, et al. Quantitative assessment of core/penumbra mismatch in acute stroke $\mathrm{CT}$ and MR perfusion imaging are strongly correlated when sufficient brain volume is imaged. Stroke 2008;39:2986-92

8. Kamalian S, Kamalian S, Konstas AA, et al. CT perfusion mean transit time maps optimally distinguish benign oligemia from true "atrisk" ischemic penumbra, but thresholds vary by postprocessing technique. AJNR Am J Neuroradiol 2012;33:545-49

9. Wintermark M, Flanders AE, Velthuis B, et al. Perfusion-CT assessment of infarct core and penumbra: receiver operating characteristic curve analysis in $\mathbf{1 3 0}$ patients suspected of acute hemispheric stroke. Stroke 2006;37:979-85

10. Kalender WA. Computed Tomography. Fundamentals, System Technology, Image Quality, Applications. New York: Wiley; 2000

11. Barrett JF, Keat N. Artifacts in CT: recognition and avoidance. $R a-$ diographics 2004;24:1679-91

12. Prokop M, Galanski M. Spiral and Multislice Computed Tomography of the Body. New York: Thieme; 2003

13. Cianfoni A, Colosimo C, Basile M, et al. Brain perfusion CT: principles, technique and clinical applications. Radiol Med 2007;112: 1225-43

14. Wintermark M, Reichhart M, Thiran JP, et al. Prognostic accuracy of cerebral blood flow measurement by perfusion computed tomography, at the time of emergency room admission, in acute stroke patients. Ann Neurol 2002;51:417-32

15. Kudo K, Sasaki M, Ogasawara K, et al. Difference in tracer delayinduced effect among deconvolution algorithms in CT perfusion analysis: quantitative evaluation with digital phantoms. Radiology 2009;251:241-49

16. Wintermark M, Albers GW, Alexandrov AV, et al. Acute stroke imaging research roadmap. Stroke 2008;39:1621-28
17. van der Schaaf I, Vonken EJ, Waaijer A, et al. Influence of partial volume on venous output and arterial input function. $A J N R A m J$ Neuroradiol 2006;27:46-50

18. Lee T, Stewart E. Scientific basis and validation. In: Cuenod CA, Miles KA, eds. Multidetector Computed Tomography in Oncology: CT Perfusion Imaging. London, UK: Informa Healthcare; 2007:15-46

19. Aviv RI, d'Esterre CD, Murphy BD, et al. Hemorrhagic transformation of ischemic stroke: prediction with CT perfusion. Radiology 2009;250:867-77

20. Konstas AA, Goldmakher GV, Lee TY, et al. Theoretic basis and technical implementations of CT perfusion in acute ischemic stroke. Part 2. Technical implementations. AJNR Am J Neuroradiol 2009;30:885-92

21. Riordan AJ, Prokop M, Viergever MA, et al. Validation of CT brain perfusion methods using a realistic dynamic head phantom. Med Phys 2011;38:3212-21

22. Dale AM, Fischl B, Sereno MI. Cortical surface-based analysis. I. Segmentation and surface reconstruction. Neuroimage 1999;9: 179-94

23. Koopmans PJ, Manniesing R, Niessen WJ, et al. MR venography of the human brain using susceptibility weighted imaging at very high field strength. MAGMA 2008;21:149-58

24. Bushberg J, Seibert J, Leidholdt E, et al. The Essential Physics of Medical Imaging. 2nd ed. Philadelphia: Lippincott, Williams \& Wilkins; 2002

25. Hoffmann KR, Nazareth DP, Miskolczi L, et al. Vessel size measurements in angiograms: a comparison of techniques. Med Phys 2002; 29:1622-33

26. Tang C, Blatter DD, Parker DL. Accuracy of phase-contrast flow measurements in the presence of partial-volume effects. J Magn Reson Imaging 1993;3:377-85

27. Lapin GD, Allen CV, Groothuis DR. Noninvasive measurement of arterial blood-plasma concentration of iodinated contrast agents from CT scans of human brain. J Comput Assist Tomogr 1994;18: 363-69

28. Mchedlishvili G, Varazashvili M, Kumsishvili T, et al. Regional hematocrit changes related to blood flow conditions in the arterial bed. Clin Hemorheol Microcirc 2003;29:71-79

29. Aspelin P. Effect of ionic and nonionic contrast-media on wholeblood viscosity. Acta Radiol Diagn (Stockh) 1978;19:977-89 